\title{
Application Research of the Moist Wound Theory in Chronic Wound Nursing
}

\author{
Shuying Ling \\ The Fifth Affiliated Hospital of Guangzhou Medical University, Guangzhou 510700, Guangdong, China \\ Email:673188907@qq.com
}

\begin{abstract}
Objective - To explore the application value of observation of moist wound theory in chronic wound care. Methods - From February 2019 to May 2020, a total of 96 patients with chronic wounds were treated in this hospital. The patients were divided into two groups based on the two-color ball method, with 48 cases in each group. Routine wound nursing (48 patients, the control group) and moist wound theory (48 patients, the experimental group) were used to compare the wound healing between the two groups. Results - The wound healing rate of 48 patients in the experimental group was significantly higher than that in the control group, and there was a significant difference $(\mathrm{P}<0.05)$. The incidence of chronic wound complications (wound pain, wound infection, tissue adhesion) of 48 patients in the experimental group was significantly lower than that in the control group, and there was a significant difference $(\mathrm{P}<0.05)$. Conclusion The application of moist wound theory in chronic wound nursing can promote the rehabilitation of patients with chronic wounds, improve the rate of wound healing, reduce the incidence of complications, and improve the patients' nursing efficacy.

Keywords: moist wound theory, routine wound nursing, chronic wound nursing, wound healing, good rate, complications
\end{abstract}

\section{Introduction}

Chronic wounds mainly refer to wounds in which skin and soft tissue defects have occurred for 14 days or more than 1 month and cannot be repaired normally. This kind of wounds are mostly secondary wounds, such as diabetic foot ulcer wounds, paraplegic wounds, etc. ${ }^{[1]}$ This type of chronic wound has the characteristics of easy infection, difficult healing, slow healing, and easy to be affected by the primary disease. For this kind of chronic wounds, many methods such as antiinfection, dressing change and debridement have been used to promote the healing of chronic wounds. However, with the continuous advancement of my country's medical level, the clinical understanding of this type of chronic wounds has gradually deepened, and moist dressings have entered the clinic, and good results have been obtained in various wound treatments. Studies ${ }^{[2-3]}$ pointed out that the application of moisturizing dressings in the treatment of chronic wounds can achieve relatively ideal results; wound healing is faster and wound healing time is shorter. The wide application of moisturizing dressings has made clinics pay more and more attention to the theory of moist wounds, and a lot of research has been conducted on this theory.

\section{Materials and methods}

\subsection{Clinical materials}

From February 2019 to May 2020, a total of 96 patients with chronic wounds were treated and treated in this hospital. The patients were divided into two groups based on the two-color ball method, with 48 cases in each group. The ratio of male to female cases in the experimental group was 27/21; the age was 22 79 years, with an average of (50.87 \pm 6.95$)$ years old. The ratio of male to female cases in the control group was 28/20; the age was $21 \sim 79$ years, with an average of $(50.49 \pm 6.87)$ years old. Compare the relevant data of the two groups of patients, and the comparison results showed no difference $(\mathrm{P}>0.05)$.

\subsection{Methods}

The 48 patients in the control group received routine wound nursing. After the patient is admitted to the hospital, the local wound of the patient is disinfected with iodophor, and the local wound is wiped with a cotton ball moistened with normal saline to remove the secretions on the surface of the wound. In addition, carry out corresponding expansion treatment to drain all the inactivated and necrotic tissues out of the wound, and then cover with sterile gauze. Combined with the actual situation of the patient's wound, guide the patient to change the dressing regularly. 
48 patients in the experimental group implemented the moist wound theory. (1) Provide a humid environment. Based on the patient's wound size, color and amount of exudation, choose a suitable occlusive dressing to apply outside the wound to provide a moist, low-oxygen environment for wound healing. The packing should not be too tight to avoid forming pressure on the wound, and hindering the normal growth of the wound and the normal operation of the microcirculation. Change the dressing regularly, and change the dressing in a sterile environment. If there is yellow rotting sediment (necrotic tissue) on the surface of the wound, it should be removed in time, and then the dressing should be replaced. The packing time and packing tightness should be reasonably controlled. (2) Choose the correct dressing. The nurse needs to choose the appropriate dressing according to the patient's wound exudate volume and the size of the wound; combined with the actual situation of the patient's local wound, decide whether to perform compression bandaging or decompression bandaging; choose dressings with different thickness and shape according to the location of the wound; choose dressings with different viscous strength combined with skin tolerance. If the exudate is greater than $10 \mathrm{ml} / 24 \mathrm{~h}$, and it is an infectious wound, apply an exudate dressing to continuously absorb the exudate so that the wound is always moist. (3) Pay attention to the general condition of the patient. During the dressing change of the patient's wound, attention should be paid to the patient's general condition. It is necessary to pay close attention to the blood biochemical test results of the patients, and timely feedback the physical feelings to the doctors to prompt the doctors to adjust the clinical medication in time. During the nursing period, attending physicians, responsible nurses and patients should participate in the nursing work together to maximize the pertinence and effectiveness of wound care. Nurses actively feed back the patient's relevant conditions, actively participate in the patient's clinical medication, combine the patient's general condition, complete the wound dressing work, so as to relieve the patient's wound pain, promote wound healing, and then improve the patient's physical and mental comfort. (4) Wound nursing records. In the process of providing wound care to patients, it is necessary to record the patient's wound nursing content in detail, such as dressing changes and wound changes. The nursing staff or the attending physician can learn about the patient's wound condition in time through the nursing records and provide follow-up services to the patient.

\subsection{Observation indicators}

(1) The excellent rate of wound healing, and the evaluation criteria of curative effect ${ }^{[4-5]}$ are as follows. Excellent: After nursing, the patient's wound healed well and the epithelial tissue completely covered the wound. Good: After nursing, the patient's wound healed well; the amount of wound exudate was significantly reduced; the growth of granulation tissue was relatively ideal; the epithelial tissue covered at least $50 \%$ of the wound surface. Medium: After nursing, the patient's wound is healed well; the amount of wound exudate is reduced; granulation tissue grows; epithelial tissue covers $25 \% \sim 50 \%$ of the wound. Poor: After nursing, the patient's wound did not heal significantly; the amount of wound exudate was not significantly reduced; the granulation tissue was almost invisible; the epithelial tissue coverage was less than $25 \%$.

(2) The incidence of chronic wound complications (wound pain, wound infection, tissue adhesion).

\subsection{Statistical methods}

The materials were analyzed by SPSS 21.0 statistical software.

\section{Results}

\subsection{Analysis of the good rate of wound healing}

The good rate of wound healing of 48 patients in the experimental group was significantly higher than that in the control group, and there was a significant difference $(\mathrm{P}<0.05)$. See Table 1 for details.

Table 1. Analysis of good rate of wound healing (n/\%)

\begin{tabular}{|c|c|c|c|c|c|c|}
\hline Groups & The number of cases & Excellent & Good & Medium & Poor & Good rate \\
\hline Experimental group & 48 & 22 & 24 & 2 & 0 & $46(95.8 \%)$ \\
\hline $\mathrm{X}^{2}$ value & - & - & - & - & - & 14.332 \\
\hline
\end{tabular}

\subsection{Analysis of the incidence of chronic wound complications}

The incidence of chronic wound complications of 48 patients in the experimental group was significantly lower than that in the control group, and there was a significant difference $(\mathrm{P}<0.05)$. See Table 2 for details. 
Table 2. Analysis of the incidence of chronic wound complications (n/\%)

\begin{tabular}{cccccc}
\hline Groups & The number of cases & Wound pain & Wound infection & Tissue adhesion & The incidence of complications \\
\hline $\begin{array}{l}\text { Experimental group } \\
\begin{array}{l}\text { Control group } \\
\text { X }\end{array}\end{array}$ & 48 & 1 & 1 & 0 & $2(4.2 \%)$ \\
$X^{2}$ value & - & 4 & - & - & $10(20.8 \%)$ \\
P value & - & - & - & - & 12.322 \\
\hline
\end{tabular}

\section{Discussion}

Chronic wounds mainly refer to wounds in which skin and soft tissue defects have occurred for 14 days or more than 1 month and cannot be repaired normally. Such wounds are mostly secondary wounds, such as diabetic foot ulcer wounds and paraplegic wounds. In the past, it was clinically believed that keeping the wound as dry as possible could effectively prevent wound infection and promote wound healing. However, with the improvement of the level of clinical medical treatment, clinical studies have clearly pointed out that the wound healing rate of patients in a moist environment is twice that in a dry environment. This view lays a solid foundation for the clinical introduction of wet dressings, and also allows the moist wound theory to be widely used in clinical practice.

The moist environment does not refer to the moist healing environment. Excessive wound exudate will cause excessive growth of granulation tissue at the wound site of the patient, which in turn will cause the skin around the wound to macerate, and eventually affect wound healing and increase the patient's physical and mental discomfort. From a medical point of view, moist wound healing mainly refers to the fact that if there is local moisturization in human wounds, it cannot promote the formation of scabs and promote wound healing ${ }^{[6]}$; but in this case, through nursing intervention, the formation of a moist environment similar to the physiological state of the human body in the wound site of the patient can promote granulation growth, skin cell division, epithelial crawling, and finally promote wound healing. However, in order to create such an ideal wet environment, it is necessary to ensure that the wound has new granulation tissue, and there will be no adhesion between the dressing and the wound tissue during the application of the dressing. Only in this way can the tissue tearing pain caused by dressing change be prevented; the wound will be damaged again; the wound healing can be accelerated ${ }^{[7]}$. Clinical studies have pointed out that the full use of moist wound theory in chronic wound care can accelerate the growth of granulation tissue in chronic wounds, promote the formation of scabs, promote wound healing, and avoid complications ${ }^{[8]}$. This research agrees with the above viewpoints. The results of this study indicate that through the application of the wet wound theory, the excellent wound healing rate of the experimental group (95.8\%) is significantly higher than that of the control group (77.1\%); the incidence of chronic wound complications $(4.2 \%)$ of the experimental group is significantly lower than that of the control group (20.8\%).

It can be seen from the above that the implementation of moist wound theory in chronic wound nursing has significant effects.

\section{References}

[1] Ma Cuifang, Fang Zheng. Research on influencing factors of knowledge about wound care of patients with chronic wounds. Chinese Health Care. 2020; 38(8): 145-146.

[2] Liu Lingling. The value of an integrated medical-care management model for chronic wound care. Electronic Journal of Practical Clinical Nursing. 2020; 5(38): 162+174.

[3] Wang Yujie. Nursing measures for chronically infected wounds of the lower extremity skin of diabetic patients. Chinese Medicine Guide. 2020; 18(24): 220-221.

[4] Lin Ruohan, Jin Lei, Qiu Jingni. The effect of applying a multidisciplinary collaborative model of chronic wounds to patients in wound care clinics. Shanxi Medical Journal. 2020; 49(15): 2069-2070.

[5] Huang Fangfang, Deng Muhong, Zheng Li, et al. Nursing care of a rare case of a rare case of deep recessed roundpit-shaped cancerous wound under the xiphoid process with wet healing theory. Journal of Nurses Training. 2017; 32(23): 2155-2156.

[6] Pang Xiaoqin. Promotion of the theory of wet healing of pressure sores in stage $\mathbb{I} \sim \mathrm{IV}$ in primary hospitals. Journal of Practical Clinical Medicine. 2017; 21(18): 220-221. 
[7] Zhang Kun. The application progress of wet dressing therapy in various wound treatments. China Medical Device Information. 2019; 25(14): 21+123.

[8] Liu Min. Application of moist healing theory in wound care of a patient with multiple venous ulcers in the lower limbs. Electronic Journal of Practical Clinical Nursing. 2019; 4(4): 88+95. 